\title{
La famille intellectuelle de Raymond Boudon
}

Raymond Boudon's intellectual family

\section{Monique Hirschhorn et Jean-Michel Morin}

\section{OpenEdition}

Journals

Édition électronique

URL : https://journals.openedition.org/ress/4317

DOI : $10.4000 /$ ress. 4317

ISSN : 1663-4446

\section{Éditeur}

Librairie Droz

\section{Édition imprimée}

Date de publication : 14 décembre 2018

Pagination : 85-104

ISSN : 0048-8046

\section{Référence électronique}

Monique Hirschhorn et Jean-Michel Morin, « La famille intellectuelle de Raymond Boudon », Revue européenne des sciences sociales [En ligne], 56-2 | 2018, mis en ligne le 14 décembre 2021, consulté le 06 janvier 2022. URL : http://journals.openedition.org/ress/4317 ; DOI : https://doi.org/10.4000/ress. 4317 


\title{
LA FAMILLE INTELLECTUELLE DE RAYMOND BOUDON
}

\author{
MONIQUE HIRSCHHORN \& JEAN-MICHEL MORIN \\ Université Paris Descartes, CERLIS \\ monique.hirschhorn@parisdescartes.fr / jean-mi.morin@wanadoo.fr
}

Résumé. «La famille intellectuelle», c'est l'expression dont se servit Raymond Boudon pour désigner dans son allocution de remerciement ceux qui venaient de contribuer en 2009 à un ouvrage d'hommage en quatre volumes. Le présent article cherche à donner un contenu à cette expression, d'abord en essayant d'identifier une partie des membres de cette famille, ensuite en s'interrogeant sur les «bonnes raisons» qui ont rendu possible l'existence de cette dernière. II vise ainsi à offrir, même si c'est d'une manière très incomplète car ce travail garde un caractère exploratoire, une représentation des effets sociaux et scientifiques que peut avoir une activité de recherche quand elle est, aussi clairement que celle de Boudon, portée par un objectif. Avec ce paradoxe: Boudon voulait avant tout que la sociologie soit reconnue comme science et, pour ce faire, il a rallié à son projet le plus grand nombre possible. Mais ce succès n'efface-t-il pas dans une certaine mesure son apport personnel?

Mots-clés: activité scientifique, école de pensée, histoire des sciences sociales, science, sociologie.

\begin{abstract}
The intellectual family" is the expression Raymond Boudon used in his acknowledgement speech to designate those who, in 2009, had just contributed to a four volumes tribute. This article seeks to give that expression substance, first by trying to identify some of the members of that family, then by questioning the "good reasons" that made its existence possible. It then offers - albeit in a very incomplete manner, since this work is still tentative-a representation of the social and scientific effects that a research activity can have when it is aimed at a definite goal, as is clearly the case with Boudon. But a paradox also arises: Boudon wanted, above all, sociology to be recognized as a science and, in order to achieve this, he rallied to his project as many members as possible. But does this success not erase his own contribution to some extent?
\end{abstract}

Keywords: history of the social sciences, school of thought, science, scientific activity, sociology. 
C'est lorsqu'on lui remit l'ouvrage d'hommage en quatre volumes, rédigé en anglais par presque une centaine de contributeurs (Cherkaoui et Hamilton 2009), que Raymond Boudon, en remerciant chaleureusement les auteurs et les personnes qui sassociaient à l'événement, parla pour la première fois de sa «famille» intellectuelle (Boudon, 2009). L'expression ne pouvait avoir qu'une connotation éminemment positive pour cet homme aussi discret quattaché aux siens. En témoignent les dédicaces de ces derniers livres: en 1986, L’Idéologie à son fils, Stéphane; en 1995, Le Juste et le Vrai à Éric, l’aîné de ses petits-fils; en 2007, les Essais sur la Théorie générale de la rationalité à sa femme qui était toujours son premier lecteur et «a joué un rôle important dans l'élaboration de (ses) écrits » (Boudon, 2003a, p. I8) ; en 20ı, La Sociologie comme science à Marc, cadet de ses petitsfils; enfin, en 20I3, son ouvrage posthume, Le Rouet de Montaigne: une théorie du croire, aux quatre dédicataires précédents ainsi qu’à sa belle-fille. Cette expression lui offrait aussi la possibilité de souligner que le groupe dont il parlait était un groupe ouvert, comme dans une vraie famille, où «chacun a sa personnalité», «tout le contraire d'une secte et même d'une école $»^{1}$, un groupe uni par l'attachement à «des valeurs et des croyances communes sur les sciences sociales ainsi que sur les responsabilités de ces dernières à l'endroit de la société » (Boudon, 2009). Il n’est toutefois jamais allé plus loin dans la présentation de sa famille intellectuelle. La prendre comme objet est donc un exercice difficile, d'abord parce cette famille, telle qu'il la conçut, ne peut être qu'une famille étendue, comprenant plusieurs générations, encore plus nombreuse que le groupe auquel il s'adressait, ce qui pose le problème de l'identification de ses membres, ensuite, parce quau-delà de l'affirmation des valeurs partagées, il faut comprendre quelle a été la nature des relations et comment elles se sont constituées.

Pour répondre à la première question, on peut, en restant dans la même logique que la sienne, constituer les listes de ceux qui ont participé ou se sont associés d'une manière ou d'une autre à son activité scientifique: celle des membres du Centre d'études sociologiques (CES) où il a été attaché, puis chargé de recherche de 196I à

I Boudon partage avec nombre de sociologues français de sa génération le refus de faire école. Sur la notion d'école en sociologie, voir Hirschhorn, 2018, et, pour une première approche de la question, voir Morin, 2006, p.56-62. 
I963 et qu'il a dirigé, à la suite de Jean Stoetzel, de 1968 à $197 \mathrm{I}^{2}$; celle des membres de son laboratoire: le Groupe d'étude des méthodes de l'analyse sociologique (GEMAS) qu'il a créé en 197I et dirigé jusqu’en $1999^{3}$ ainsi que celle du comité de rédaction de L’Année sociologique qu'il a présidé de 1978 à 2002, y compris après son départ de ces deux institutions ${ }^{4}$; celle des étudiants dont il a dirigé la thèse, des rédacteurs d'entrées dans les dictionnaires qu'il a co-dirigés (Boudon et al., 1989; Borlandi et al., 2005), dans l'encyclopédie (Smelser et Baltes, 200I) dont il a dirigé une partie, des auteurs publiés dans la collection «Sociologies» qu'il créé en 1977 avec François Bourricaud en 1977 aux PUF (Langlois, 2008, p. 349-359), des contributeurs à des ouvrages qui lui ont été consacrés (Busino et Valade, 1998; Baechler, Chazel et Kamrane, 2000; Cherkaoui et Hamilton, 2009), des collègues qui l'ont élu à titre étranger dans leurs académies (British Academy, Société royale du Canada, Académie des sciences humaines de Saint Pétersbourg, Académie des arts et sciences d'Europe centrale), des membres de l'European Academy of Sociology dont il a été le premier président, de l'Académie des sciences morales et politiques qui l'ont élu en 1990; des souscripteurs pour son épée lors de son élection... On le voit, le nombre et la longueur de ces listes fait de l'identification des membres une mission quasi impossible. Au mieux, l'on peut espérer parvenir à donner une image à peu près fidèle de la composition de la famille, étant entendu que ne pas être mentionné ne signifie pas que l'on n’appartienne pas à la familles.

La seconde question est celle de la nature des relations. Dans la vision très positive que Boudon avait de la famille, il était entendu que ces relations étaient fructueuses. Mais cela ne signifie pas qu’elles aient eu toutes la même intensité et le même contenu. À l'évidence, il y a eu des liens forts fondés sur des échanges,

2 Sur le CES, voir la thèse de Patricia Vannier (1999).

3 Le GEMAS a fusionné depuis janvier 2010 avec le CESS (Centre d'études sociologiques de la Sorbonne) et est devenu le GEMASS (Groupe d'étude des méthodes de l'analyse sociologique de la Sorbonne). Pour éviter toute méprise, il sera le plus souvent nommé «le laboratoire».

4 Les directeurs ultérieurs du laboratoire, les présidents ultérieurs de L'Année, ainsi que la plupart des membres arrivés après son départ, font partie à des titres divers de la famille.

5 Nous présentons par avance nos excuses à ceux que nous n'aurons pas mentionnés comme à ceux qui pourraient s'offusquer de l'être, la conception qu'avait Boudon de la famille intellectuelle étant fort englobante. 
des collaborations, des réalisations communes, s'inscrivant le plus souvent dans la durée. Il y a eu aussi des liens plus faibles, se traduisant par des manifestations, des contributions ponctuelles et ce n'est probablement pas trahir sa pensée que de présenter les membres de sa famille en distinguant les «proches» des «alliés» et en situant les membres de chacune de ses catégories au regard de la position qu'ils ont occupée par rapport à lui. Cela conduit, si l'on file la métaphore familiale, à distinguer dans les proches, les maîtres, les pairs, les élèves, et dans les alliés, les Français des étrangers des membres d’autres disciplines. On peut donc, en se servant de cette grille, reprendre les listes mentionnées précédemment tout en gardant à l'esprit que ce travail a une dimension exploratoire, qu'une recherche plus approfondie supposerait d'exploiter les $\operatorname{archives}^{6}$ et de procéder à une véritable enquête auprès de ceux qui ont connu Boudon.

\section{LES PROCHES (MAÎTRES, PAIRS, ÉLÈVES)}

I.I. LES MAITTRES

De tous les membres de la famille, ce sont assurément les maîtres qui sont les plus faciles à identifier, car l'on dispose pour les identifier d'un certain nombre de textes dans lesquels Boudon a présenté son autobiographie intellectuelle et tout particulièrement ses années de formation (Boudon, 200 ; Boudon, 2003a; Boudon, 2010; Boudon, 2013). Après être rentré à l’École normale supérieure en 1955 et avoir passé l’agrégation de philosophie en 1958, Boudon se trouva confronté au choix du sujet d'une thèse et de son directeur. Tenté par la sociologie qui lui paraissait être «une sorte de philosophie qui s'intéresserait au monde moderne, au monde concret» (Boudon, 20I3, p. 343), hésitant encore entre la sociologie et l'économie appliquée, dont il avait découvert l'intérêt à travers Georges Théodule Guilbaud, attiré par les mathématiques (il avait suivi les cours de Marc Barbut à l'institut Henri-Poincaré), il partit en stage, grâce à Raymond Aron dont il avait beaucoup apprécié le cours sur

6 Pour une étude systématique, les historiens disposent désormais du Fonds Raymond Boudon, constitué par les archives léguées par Boudon en 2012 et inventoriées en 2014, sous la coordination de Brigitte Mazon, responsable du service des archives de l'EHESS. L'inventaire est téléchargeable sur le site du GEMASS. 
Montesquieu, de 1960 à 196I à Columbia où la rencontre avec Paul Lazarsfeld, «le sociologue contemporain qu'il a le plus admiré » (Boudon, 200I, p. 2I), fut décisive. Il lui fournit le sujet de sa thèse principale : un bilan de l'apport de la pensée mathématique aux sciences sociales. De retour à Paris, il prit comme directeur de thèse celui dont il se sentait le plus proche intellectuellement, Jean Stœetzel, titulaire de la chaire de psychologie sociale nouvellement créée, directeur du CES, et qui, à travers l'Institut français d'opinion publique (IFOP), développait une sociologie fondée sur les enquêtes quantitatives et, comme directeur de sa thèse secondaire sur l'idée de structure, Raymond Aron. Les deux thèses seront très vite publiées: L'Analyse mathématique des faits sociaux en 1967, À quoi sert la notion de structure? Essai sur la signification de la notion de structure en 1968. Entre temps, il édita, en collaboration avec Lazarsfeld, lors du séjour de ce dernier à Paris, trois recueils de textes sur la méthodologie quantitative (Boudon et Lazarsfeld, I965; 1966; Boudon, Lazarfeld et Chazel, 1969). Il aura eu ainsi deux maîtres: Lazarsfeld, dont l'influence sur sa pensée a été essentielle et Stœetzel, qui l'accompagnera tout au long de sa carrière.

En a-t-il eu d'autres? Il ne semble pas. S'il manifesta un rejet très explicite de Georges Gurvitch, qui aurait pu être l'un de ses maîtres, mais dont l'hyperempirisme dialectique lui paraissait sans prise sur la réalité, il avait en revanche une grande admiration pour Aron, mais il voyait en lui moins un sociologue qu'un très grand intellectuel. Il avait également été fort impressionné par les capacités théoriques de Robert Merton, comme par une partie de ses collègues américains. Mais il n’en fit pas des maîtres. À Paris, au début des années 1960, il eut plutôt des relations privilégiées avec des collègues un peu plus âgés que lui, qui jouèrent alors le rôle de mentors: Alain Touraine, qui lui confia la réalisation d'un rapport d'enquête sur les ouvriers d'origine agricole et qui sera toujours présent dans les grandes occasions comme la remise de l'épée à l'Académie ou les hommages : André Davidovitch, le criminologue, qui lui permit de réaliser sa première grande enquête sur les statistiques judiciaires d'abandon de poursuite des juges; Mattei Dogan, le politologue; Henri Mendras dont il partageait la même volonté de développer une sociologie appliquée et avec qui il aura par la suite de nombreux échanges ; 
Viviane Isambert-Jamati, spécialiste de l'enseignement, qu'il retrouvera un peu plus tard à L’Année sociologique; François Isambert, sociologue de la religion, Henri Jamous, spécialiste des échelles d’attitude. L’autre grande figure, entre maître et mentor, a été celle de Jean Cazeneuve, le futur directeur de la télévision française, qui le recrutera un peu plus tard à la Sorbonne et qui, membre de l’Académie des sciences morales et politiques, lui remettra son épée d’académicien en le qualifiant «d'empêcheur de penser en rond» (Cazeneuve, I99I, p. I7).

Toutefois, en restant dans la recherche des maîtres, ce n'est pas trahir sa pensée que d'y inclure les sociologues classiques, ceux qu'il n’aura rencontrés qu'à travers leurs écrits. La relation qu'il a avec eux, comme le souligne Michel Dubois (2000, p. 16-19), n'est pas celle distanciée d'un historien des idées qui essaie de comprendre l'unité d'une pensée ou d'un historien de la sociologie qui tente de la resituer dans son contexte, mais une relation fondée sur une sympathie intellectuelle qui l'autorise à dialoguer avec eux, à se saisir de leurs travaux pour conforter sa propre pensée (Boudon, 1998-2000). De ce fait, ils n'occupent pas tous la même place. S'il attache une place à part à Simmel dont il a traduit en 1984 Les Problèmes de la philosophie de l'histoire, s'il porte beaucoup d'intérêt à Durkheim, ne serait-ce que pour montrer que ce dernier fut infidèle, heureusement de son point de vue, aux Règles de la méthode sociologique, ses vrais maîtres sont Weber et surtout l'indépassable Tocqueville, le penseur français le plus original, celui qui nous aide à comprendre les sociétés modernes.

\section{I.2. LES PAIRS}

Après les maîtres, viennent les pairs ${ }^{7}$ qui vont devenir des proches. Il n'est pas difficile de les identifier, car à quelques exceptions près, qui tiennent au début de la carrière de Boudon, ils ont été ou ils sont membres permanents ou associés du laboratoire et/ou de L’Année sociologique, le plus souvent publiés dans la collection «Sociologies». Mais on ne saurait se contenter de reprendre la liste de ceux-ci, non seulement parce qu'ils sont trop nombreux, mais parce que le laboratoire comme L’Année ayant été des espaces ouverts, accueillant des

7 Ce qui ne veut pas dire que tous les pairs ont le même statut académique. La parité est entendue d'un point de vue intellectuel. 
universitaires et des chercheurs aux profils fort différents, certains ne peuvent être que des alliés. On ne retiendra donc ici que des proches ayant eu des collaborations effectives, le plus souvent inscrites dans la durée.

Si l'on suit quelque peu l'ordre chronologique, il y a d'abord son condisciple, Jacques Lautman, qu'il a rencontré à l'École normale supérieure et auquel l’a lié une indéfectible amitié. Admirateur comme lui de Stœtzel, il a joué un rôle majeur dans le développement des sciences sociales au Centre national de la recherche scientifique (CNRS), suivra Boudon à L'Année et participera à tous les hommages. Comment ne pas citer ensuite, François Bourricaud, de I2 ans son aîné, qui le rejoignit en 1970 à la Sorbonne. Spécialiste français de Parsons, cofondateur de la collection «Sociologies», co-auteur du Dictionnaire critique de la sociologie publié en 1982 aux PUF, il a été assurément le plus proche autant d'un point de vue amical qu'intellectuel; puis, François Chazel, analyste des processus politiques et sociaux, co-auteur du Traité (Boudon, 1992), co-directeur du premier livre d'hommage (Baechler, Chazel et Kamrane, 2000) ; Bernard Valade, historien des idées et spécialiste de Pareto, co-directeur du Dictionnaire de la pensée sociologique (Borlandi et al., 2005), co-auteur du Traité, son successeur en 2002 à la présidence de L’Année; Jean Baechler, spécialiste de sociologie historique, élu comme lui à l'Académie des sciences morales et politiques, co-auteur du Traité (Boudon, 1992), co-directeur du premier livre d’hommage (2000) ; Bernard-Pierre Lécuyer, historien de la sociologie, directeur de recherche au CNRS, co-directeur du premier dictionnaire (Boudon et al., 1989) ; Philippe Besnard, directeur de la Revue française de Sociologie et fondateur du Groupe d'études durkheimiennes, co-directeur du premier dictionnaire (Boudon et al., 1989), trop tôt disparu, dont Boudon (2003b) écrivit qu'il constituait pour la sociologie française «un repère intellectuel»; Massimo Borlandi, historien de la sociologie, co-directeur du Dictionnaire de la pensée sociologique (Borlandi et al., 2005), Charles-Henri Cuin, spécialiste de la mobilité sociale et d'histoire de la sociologie; Peter Hamilton, co-directeur avec Mohamed Cherkaoui de l'ouvrage d'hommage en quatre volumes (2009); Peter Hedström, figure clé de la sociologie analytique à laquelle Boudon a adhéré8,

8 Voir, entre autres, la contribution de Boudon à l'ouvrage dirigé par Pierre Demeulenaere, Analytical Sociology and Social Mechanisms (2011). 
Michel Forsé, spécialiste du changement social, et, si l'on passe à la génération suivante, Jean-Christophe Marcel, historien de la sociologie; Gérald Bronner dont la sociologie des croyances étayée par les sciences cognitives s’inscrit dans le prolongement des travaux de Boudon, Gianluca Manzo qui travaille sur la rationalité de l'acteur et les inégalités, etc. Déjà longue, cette liste est incomplète, ne serait-ce que parce qu'elle laisse de côté des relations plus anciennes, liées à un commun intérêt pour l'application des mathématiques aux sciences sociales, celle avec Guy Michelat, connu en classe terminale à Condorcet et retrouvé lors de son service militaire dans la Marine nationale; avec Daniel Derivry qu'il rencontra au CES et qui le suivit au GEMAS; avec Jean-Paul Grémy qui a collaboré à son ouvrage sur Les Mathématiques en sociologie (Boudon, 1971a) et qui a travaillé avec lui sur des modèles de simulation; avec Pierre Parlebas, son collègue de Paris V, modélisateur des jeux sportifs. Et surtout il manque ceux dont la proximité repose initialement sur le choix de prendre Boudon comme «maître», les anciens élèves.

\subsection{LES ÉLÈVES}

C'est un groupe dont l'importance est considérable (entre I970 et 2006, Boudon a fait soutenir 80 thèses tous régimes confondus), car Boudon a prêté une très grande attention à ses élèves, au moment de leur thèse, qu'il dirigeait en leur accordant beaucoup de liberté de pensée sous condition que l'exigence scientifique soit maintenue, mais aussi dans leur carrière ultérieure. Si certains, comme Philippe Cibois ou Claude Dubar se sont éloignés, la plupart d'entre eux ont gardé un lien fort qui, comme précédemment, s'exprime par la participation au laboratoire en tant que membre permanent ou associé et/ou à la revue, ainsi que, le plus souvent par la publication d'un de leurs travaux dans la collection. Un premier nom s'impose immédiatement : celui de Mohamed Cherkaoui, qui prendra la direction du GEMAS après le départ de Boudon. Spécialiste des systèmes d'enseignement, des questions d'inégalités et de mobilité sociale, il sera co-directeur de nombreuses entreprises éditoriales collectives: deux dictionnaires (Boudon et al., 1989; Borlandi et al., 2005), trois ouvrages publiés chez Sage : un recueil de textes classiques, The Classical Tradition in Sociology (Boudon et al., 1997), une anthologie en 4 volumes des recherches sociologiques, Central Currents in 
Sociological Theory (Boudon et Cherkaoui, 1999) et une présentation de la tradition européenne en méthodologie qualitative, The European Tradition in Qualitative Research (Boudon et al., 2003). Co-auteur du Traité, contributeur aux ouvrages collectifs consacrés à Boudon, il co-dirigera avec Peter Hamilton l'ouvrage d'hommage en quatre volumes (2009). On trouve aussi Terry Shinn en sociologie de la science; Jean-Gustave Padioleau sur la sociologie de l'ordre social et des médias, Simon Langlois, analyste du changement social et de la consommation. Dans une relation moins étroite, Yves Grafmeyer qui a travaillé sur la Banque et la sociologie urbaine, Monique Hirschhorn, sur l'éducation et l'histoire de la sociologie, qui invita Boudon comme conférencier au congrès de l'Association internationale des sociologues de langue française (AISLF) à Evora en 1996.

À la génération suivante, Pierre Demeulenaere, dont les travaux sur la rationalité et les normes prolongent ceux de Boudon. Elu professeur à la Sorbonne et président de L’Année à la suite de Bernard Valade, il a succédé en 2018 à Olivier Galland à la direction du GEMASS, et recueille ainsi une grande partie de l'héritage institutionnel; Nathalie Bulle, spécialiste de la sociologie de l'éducation; Sylvie Mesure, spécialiste de l'histoire et de l'épistémologie des sciences sociales, dont l'activité éditoriale a contribué à la diffusion de la conception de la sociologie défendue par Boudon; Lilyane Deroche-Gurcel, spécialiste de Georg Simmel; Michel Dubois en sociologie des sciences et en histoire de la sociologie, qui a rédigé l'un des premiers ouvrages de synthèse sur l'œuvre de Boudon (Dubois, 2000) ; Dominique Guillo en sociologie de la connaissance scientifique; Guillaume Erner, analyste de l'antisémitisme, journaliste de radio qui a obtenu qu'il soit fait mention du décès de Raymond Boudon en 2013 à l’antenne de France-Inter...

Mais il faut aussi citer ceux qui sont restés proches, bien qu'ils n'ont pas ou n'ont plus d’attaches institutionnelles avec le laboratoire ou L’Année, comme Salvator Abbruzzese, sociologue italien de la religion, Alban Bouvier qui a dirigé en 1997 avec Chazel et Boudon un ouvrage sur la dimension cognitive dans l'analyse sociologique; Hervé Dumez, dont la thèse a porté sur Walras et qui travaille sur le management et l'innovation; Louis-André Vallet, spécialiste de la mobilité sociale, sollicité pour l'enquête Commentaire de 20 I, Philippe Nemo, plus philosophe que sociologue, théoricien de la pensée libérale en politique 
et éducation; Annette Disselkamp qui a travaillé sur Weber et la sociologie des valeurs; Emmanuelle Betton sur le sentiment de justice; Renaud Fillieule, qui a collaboré avec Boudon en 2002 pour une nouvelle édition, revue des deux tiers, du «Que sais-je? » sur Les Méthodes en sociologie que Boudon avait publié en I969; Jean-Michel Morin, auteur en 2006 d'un livre sur Boudon, et parmi ses derniers élèves, Pascal Sanchez qui a travaillé sur les croyances collectives ainsi que Razmig Keucheyan qui, après avoir mis en question les théories constructivistes s'intéresse aux nouvelles pensées critiques.

À cette liste de proches, dont on doit à nouveau redire combien elle est incomplète, il faut adjoindre les noms de quatre personnes qui ont joué dans la vie intellectuelle de Boudon un rôle décisif. Ce sont les deux éditeurs qui lui ont toujours fait une totale confiance: Michel Prigent aux PUF et Claude Durand chez Fayard; le directeur de la revue Commentaire dans laquelle il a beaucoup publié, Jean-Claude Casanova, auquel on peut ajouter, bien qu'il ait appartenu à la sphère académique, Giovanni Busino qui, comme éditeur à la Librairie Droz et directeur de la Revue européenne des sciences sociales lui a toujours apporté un très fort soutien: dès 197 I avec la publication chez Droz dans sa collection d'un recueil d’articles, La Crise de la sociologie (Boudon, 197Ib), et en 1998 en co-dirigeant avec Bernard Valade un numéro de la Revue européenne des sciences sociales sur «la valeur de la sociologie de Raymond Boudon». Enfin, last but not least, Annie Devinant, sociologue et auteur d'une présentation des courants de la pensée sociologique par les textes (Devinant, 1999), assistante fidèle de Boudon, remerciée à maintes reprises pour sa relecture attentive et constructive des manuscrits, cumulant de 2007 à 2017 les fonctions de secrétaire de rédaction et de secrétaire générale de L’Année sociologique.

\section{LES ALLIÉS (SOCIOLOGUES FRANÇAIS, SOCIOLOGUES ÉTRANGERS, MEMBRES D'AUTRES DISCIPLINES)}

Il reste maintenant à identifier les membres de la famille dont le lien avec Boudon n’a pas impliqué, comme précédemment, une collaboration ou une filiation directe et revendiquée, seulement une entente intellectuelle s'exprimant de diverses manières tout en tenant compte que, pour certains, la distinction 
entre proche et allié n'est pas toujours évidente et peut ne pas correspondre à la représentation que l'intéressé se fait de son positionnement. On les trouve aux côtés des proches dans les listes mentionnées précédemment: celle des contributeurs aux ouvrages collectifs, à l'enquête de Commentaire «La sociologie : science ou discipline? » dirigée par Boudon en 201 I, aux ouvrages d'hommage et, en particulier, celle des collègues étrangers qui l'ont invité, qui l'ont élu dans leurs académies, celle de ses électeurs à l'Académie des sciences morales et politique, celle des souscripteurs pour l'épée, celle des rédacteurs des éloges lors de sa disparition. La tâche est encore plus délicate que lorsqu'il s'agit d'identifier les proches. Pourtant, il est impossible de ne pas les inclure dans la famille, car c'est en majorité à des alliés que Boudon s'était adressé lors de l’allocution où il avait utilisé le terme de famille. À la lecture de ces listes, trois constats s'imposent immédiatement : l'importance du nombre de sociologues étrangers; la présence de sociologues français couvrant les diverses spécialités de la discipline et occupant des positions significatives; la présence de membres d’autres disciplines.

\section{I. LES SOCIOLOGUES FRANÇAIS}

En sociologie du changement social, Henri Mendras, déjà cité ; en sociologie de l'action, Alain Touraine, déjà cité ; en sociologie de l'action publique, Patrice Duran, membre de L'Année à qui l'on doit d'avoir suggéré à Boudon d'écrire La Sociologie comme science (Boudon, 20ı); en sociologie de l'éducation, Vivianne Isambert-Jamati, déjà citée également, Marie Duru-Bellat qui ont été l'une et l'autre membres de L’Année; en sociologie du travail et des organisations: Jean-Daniel Raynaud, Françoise Piotet qui a été membre de L’Année, Michel Crozier, membre de l’Académie des sciences morales et politique, Erhard Friedberg, co-auteur du Traité; en sociologie politique: Dominique Schnapper, Pierre Birnbaum, co-auteur du Traité, Philippe Bénéton qui a été membre de L’Année; en sociologie des valeurs: Patrick Pharo; en épistémologie: Jean-Michel Berthelot; en sociologie des médias: Francis Balle, co-auteur du Traité; en sociologie et démographie: Michèle Dion, membre de L’Année, mais aussi François Héran, professeur au Collège de France sur la chaire «Migrations et sociétés», qui fait partie de ceux 
qui ont rendu hommage à Boudon lors de son décès (Héran, 2013) ${ }^{9}$; au GEMASS, pour la période actuelle, en sociologie des inégalités: Olivier Galland, qui a dirigé le laboratoire après Mohamed Cherkaoui et Yannick Lemel; en sociologie économique: Philippe Steiner; en sociologie de l'art: Alain Quemin, etc. Une fois de plus, cette seule énumération est trop brève. Mais elle suffit à donner une idée de la place qu’a occupée Boudon dans la sociologie française.

\subsection{LES SOCIOLOGUES ÉTRANGERS}

Une des caractéristiques majeures de la famille intellectuelle de Boudon est d'être ouverte sur le reste du monde. Cela est visible lorsque l'on regarde l'appartenance nationale des membres du laboratoire et de la revue, encore plus, celle des élèves, des auteurs publiés dans la collection «Sociologies» où les traductions sont nombreuses, des contributeurs aux différents ouvrages. Cette composition trouve son explication dans les relations qu'il a nouées avec des collègues étrangers dès ses années de formation lors de ses séjours en Allemagne et aux États-Unis, puis tout au long de sa carrière en acceptant de très nombreuses invitations de collègues étrangers (il eut même à plusieurs reprises des offres de poste aux États-Unis qu'il refusa) et qui ont conduit à son élection dans plusieurs académies étrangères. Mais, de façon plus essentielle, elle est liée à la conviction qu'une science n'est pas liée à un contexte national. L'enquête qu'il a menée pour la revue Commentaire (Boudon, 20I I) pour montrer quelle était actuellement la conception que les sociologues se faisaient de la sociologie en constitue un exemple particulièrement probant. Sur 26 experts consultés sur la place de la sociologie, dix sont français - dont Boudon qui synthétise les réponses -, cinq sont allemands, deux sont anglais et deux viennent des Pays-Bas, un est espagnol, un italien, un norvégien, un suédois, un tchèque, un suisse et un canadien.

Qui sont ces alliés étrangers? Pour les évoquer, en réunissant une fois encore les contributeurs aux hommages, les souscripteurs pour l'épée, les auteurs publiés, le plus simple est de procéder par pays. En Allemagne: Hans Albert, Jürgen Friedrichs, Renate Mayntz, Birgitta Nedelmann, etc.; en Belgique:

9 La plupart des hommages exprimés lors du décès de Boudon sont consultables sur le site du GEMASS. 
Philippe Van Parijs, Claude Javeau, etc. ; au Brésil : Fernando Henrique Cardoso, José Guilherme Merquior, etc.; au Canada: Maurice Cusson, co-auteur du Traité; aux États-Unis: Daniel Bell, James Coleman, Anthony Oberschall, Seymour Martin Lipset, Stephen Luckes, Neil J. Smelser, Paul P. Bates, Thomas Schelling, etc.; en Espagne: Victor Perez-Diaz, etc.; en Grèce: Chrysostomos Mantzavinos; en Italie: Dario Antiseri, Enzo Di Nuscio, Angelo Petroni, Riccardo Viale (co-directeur de l'ouvrage collectif, L’Explication des normes sociales, PUF, 200I) ; en Israël : Shmuel Eisenstadt, Eliezer Ben Rafaël, etc. ; aux Pays-Bas: Rod Aya, Siegwart Lindenberg, Vincent Buskens; au Québec: Guy Rocher, Maurice Cusson (qui participe au Traité), Mario Bunge, Denis Szabo (criminologue d'origine hongroise, dédicataire du Déclin de la morale), etc.; en Norvège: John Elster, Arne Mastekaasa, Nathalie Rogoff-Ramsoÿ, etc.; au Royaume-Uni: Diego Gambetta, John Goldthorpe, Stein Ringen, Ernest Gellner, Peter Abell, etc. ; en Suède: Richard Swedberg, Guy Hernes, Michael Tählin, etc.; en Suisse: Giovanni Busino déjà cité, Jean Kellerhals, qui fut membre de L’Année, Pierre Moessinger, Jacques Coenen-Huther, etc. ; en Tunisie : Ali Rhida Chennoufi, etc.

\subsection{LES MEMBRES D'AUTRES DISCIPLINES}

Un autre élargissement consiste à traverser d’autres frontières, disciplinaires cette fois. Il est indéniable que la Sorbonne, les Presses universitaires de France, la Maison des sciences de l'homme de Paris, l’Année sociologique, l’Académie des sciences morales et politiques, ont offert des occasions de rencontres fructueuses, voire chaleureuses, avec des historiens: Jean Tulard, Pierre Chaunu, François Furet, Alfred Grosser, etc.; des philosophes: Maurice Clavelin, Bertrand Saint-Sernin, etc. ; un historien des mathématiques : Marc Barbut ; des démographes : Alain Girard, Jacques Dupâquier, le fondateur de la démographie historique; un anthropologue: Claude Rivière; des juristes: Jean Carbonnier fut un des présidents de L’Année, Marie-Anne Frison-Roche; voire même des économistes, comme Maurice Allais, l'individualisme méthodologique étant beaucoup moins étranger à leurs modes de pensée que les formes jugées trop littéraires de la pensée sociologique. 


\section{DES (BONNES) RAISONS D'APPARTENIR À LA FAMILLE INTELLECTUELLE}

De cette rapide et incomplète investigation ressortent plusieurs constats. La famille intellectuelle est une famille étendue et hétérogène aussi bien du fait de l'âge que des positions statutaires, des nationalités, de l'appartenance disciplinaire, des thématiques et, même, cela est plus troublant, pour une part, des orientations théoriques. Il est ainsi manifeste que Jean Baechler et Bernard Valade, deux proches, ont à l'évidence, l'un parce qu'il fait de la sociologie historique, l'autre parce qu'il est historien des idées, une autre vision que la sienne des sciences sociales. De fait, les contributions dans les livres collectifs qui ont été consacrés à Boudon partent parfois dans des directions différentes de celles qu’il privilégiait. C'est donc au pied de la lettre qu'il faut prendre la phrase qu'il a prononcé dans son allocution: «Dans une famille, chacun a sa personnalité». Boudon en était parfaitement conscient, au point d’aller jusqu'à reconnaître que certains de ses membres étaient moins convaincus que lui de la possibilité pour les sciences sociales d'être des sciences comme les autres, nacceptaient pas sa lecture des textes des pères fondateurs ou encore son refus de justifier le relativisme par la diversité des sociétés dans l'espace et le temps (Boudon, 2009).

\section{I. LA SOCIOLOGIE COMME SCIENCE}

Comment expliquer alors que cette famille intellectuelle ait pu exister, ainsi qu’en témoignent les nombreux hommages qu’a reçus Boudon de son vivant et les entreprises collectives qu'il a pu mener? Une façon de répondre est de se servir d'un des raisonnements privilégiés de Boudon, de voir dans la famille intellectuelle un effet émergent de rencontres individuelles ainsi que de projets collectifs, inscrits le plus souvent dans des structures institutionnelles (départements d'université, laboratoires, académies), et portés par quelques bonnes raisons. La première des raisons, assurément la plus forte, c'est la croyance partagée que la sociologie doit être utile à la société et qu'elle ne peut l'être que si elle se montre capable de fournir des explications solides face aux énigmes que constituent une partie des phénomènes sociaux, ce qui suppose d'y voir la combinaison d'actions individuelles rationnelles (Boudon, 20Io). Sur cette 
première raison, Boudon a beaucoup écrit. S'il a réussi à imposer sa conception, c'est qu'il n’a jamais changé d'objectif. Celui-ci est présent dès sa thèse, dans le choix de faire de la sociologie plutôt que de la philosophie, de travailler avec Lazarsfeld et Stœtzel, et on la trouve formulée très explicitement en 1971 dans l'introduction de La Crise de la sociologie. Toutes ses recherches sur l'inégalité des chances, le désordre social, le fondement des valeurs iront dans le même sens : montrer la capacité explicative de la sociologie, de même que son activité éditoriale, qui était de rendre le plus visible possible tout ce qui, dans la production sociologique présente et passée, relevait d'une véritable démarche scientifique.

Toutefois, ce n'est pas seulement l'exigence scientifique qui a soudé la famille intellectuelle. Toute démarche scientifique conduit à vérifier mais aussi à réfuter. On retrouve donc chez Boudon et la plupart de ses maîtres, proches, élèves ou alliés une propension à vouloir réfuter les idées fragiles, douteuses, fausses. Boudon a conduit lui-même cette démarche avec beaucoup de pugnacité, l'estimant particulièrement utile en France où, grâce à un terrain favorable, elles ont proliféré. C'est au point qu'une sorte d’assemblage du structuralisme, du marxisme, du sociologisme ou encore du relativisme a pu être nommé dans le monde la French Theory.

\subsection{POUR UNE SOCIÉTÉ OUVERTE}

Mais, et c'est la troisième raison qui explique l'attractivité de la famille intellectuelle, cette démarche de vérifications et de réfutations ne s'est jamais accompagnée d'une clôture quelconque. Le libéralisme philosophique, l'engagement en faveur d'une société ouverte, lui semblait indissociable de l'exigence scientifique. Il n’a donc jamais eu l'objectif de créer quelque chose qui pouvait ressembler de près ou de loin à l'idéal-type de l'école avec un chef de file, une doctrine et des disciples. La liberté de pensée qu'il reconnaissait à ses doctorants existait tout autant à l'intérieur du laboratoire qui fonctionnait, si l'on en croit le témoignage de Mohamed Cherkaoui, comme «un club de gentlemen » et où la vie scientifique était aussi agréable que possible, qu’à L’Année sociologique où il n'y avait pas à proprement parler de ligne éditoriale, simplement la volonté de ne publier que des textes dont la qualité scientifique ne puisse être discutée. Et les successeurs de Boudon, tant au laboratoire qu'à la revue, ont reconduit ce 
choix. Enfin il y a une dernière raison à la cohésion de la famille intellectuelle qui tient à la personnalité même de Boudon. Tous les témoignages concordent. Il n’a pas été un scientifique austère, mais un homme chaleureux et généreux, capable à la fois de défendre avec pugnacité ses idées et d’accepter qu'on le contredise, d’avoir des certitudes et de douter. Attitudes peu fréquentes dans le monde intellectuel qui manifestaient une personnalité plus complexe qu'elle ne le paraissait et surtout une profonde honnêteté intellectuelle. La valeur des idées lui importait plus que son succès personnel.

La famille intellectuelle est ainsi très ouverte. Ont pu même s'y rattacher des indignés, comme Judith Lazar, sociologue de la communication, qui, refusant le rejet de la pensée de Boudon dans certains cercles intellectuels parisiens, écrit un article sur le sujet et organise un grand colloque pour les étudiants internationaux de la Cité universitaire à Paris «au tournant de l'an 2000». Reste à savoir si cette famille intellectuelle continue à exister. Bien que du vivant de Boudon, outre les quatre volumes d’hommages précédemment cités, un assez grand nombre de travaux aient été consacrés à sa pensée (Di Nuoscio, I996; Busino et Valade, 1998; Assogba, 1999; Baechler, Chazel et Kamrane, 2000; Dubois, 2000; Hamlin, 2002 ; Vautier, 2002; Morin, 2006 ; Scoppettuolo, 20Io), la production, au regard de ce qui se passe pour d'autres penseurs, semble actuellement bien discrète. On peut le regretter, mais aussi en faire une autre lecture. Boudon ne serait-il pas en quelque sorte victime de sa réussite? L’idée que la sociologie peut être une véritable science ayant réussi à s'imposer, les idées fausses reculant, il ne serait plus aussi nécessaire de se rassembler pour défendre la première et combattre les dernières. C'est ce que l'on pouvait penser, en analysant les résultats de l'enquête de Commentaire de 20I I, que Boudon avait justement suscitée pour savoir ce qu'il en était. Venus d'horizons très divers, les sociologues consultés, qui, pour certains d'entre eux à l'évidence n’appartenaient pas à la «famille», comme Jean-Claude Passeron et François Dubet, se retrouvaient pour affirmer que la sociologie doit être utile et qu'elle ne peut l'être qu'en essayant d'être scientifique. 
Presque huit ans ont passé et, si l'on en juge la polémique qu’a suscité le livre de Gérald Bronner et Étienne Gehin, Le Danger sociologique, publié en 20I7, dans lequel ils dénoncent le maintien d'une conception rigide de la sociologie qui fait des comportements individuels le produit de déterminismes sociaux, le consensus qui semblait se dessiner autour de la sociologie comme science semble ébranlé. Le projet de Boudon est donc toujours d'actualité alors qu'il n’y a probablement personne pour le porter avec l'énergie qui fut la sienne. Mais ses livres sont là, réédités à de multiples reprises, traduits en plusieurs langues. Le dernier, publié de son vivant en 2012, Croire et savoir, penser le politique, la morale et le religieux, vient de paraître en coréen ${ }^{10}$. Bien qu'il soit toujours hasardeux de savoir ce qu'il adviendra d'une ouvre, il y a de fortes chances pour que celle de Boudon devienne pour la sociologie une tradition, c'est-à-dire, selon Randal Collins (1995, p. I I), ce dont on se souvient et ce à quoi l'on se réfère.

\section{BIBLIOGRAPHIE}

ASSOGBA Y, 1999, La Sociologie de Raymond Boudon, Paris/Québec, L'Harmattan/PUL. BAECHLER J, CHAZEL Fr. et KAMRANE R., 2000, L'Acteur et ses raisons. Mélanges en l'honneur de Raymond Boudon, Paris, PUF.

BORLANDI M., BOUDON R., CHERKAOUI M. et VALADE B. (dir.), 2005, Dictionnaire de la pensée sociologique, Paris, PUF.

BOUDON R., 1967, L'Analyse mathématique des faits sociaux, Thèse principale pour le doctorat d'État, Paris, Plon.

-, 1968, À quoi sert la notion de structure? Essai de signification sur la notion de structure, Thèse complémentaire pour le doctorat d'État, Paris, Gallimard.

-, 1971a, Les Mathématiques en sociologie, avec la collaboration de J.-P. Grémy, Paris, PUF.

-, 197Ib, La Crise de la sociologie. Questions d'épistémologie sociologique, Genève, Droz. -, (dir.), 1992, Traité de sociologie, Paris, PUF.

10 Traduit par Ji Young Lim, repartie dans son pays après avoir soutenu son doctorat à Paris. 
-, 200I, «Pourquoi devenir sociologue? Réflexions et évocations», Revue européenne des sciences sociales, $X X X \mid X-120$, p.5-30.

-, 2003a, Y a-t il encore une sociologie? Entretiens avec Robert Leroux, Paris, Odile Jacob.

-, 2003b, «Philippe Besnard (1942-2003)», Revue française de sociologie, 44-4, p. I.

-, 2009, Allocution de remerciements à l'occasion de la remise de l'ouvrage de Mélanges (83 articles) en son hommage, in CHERKAOUI et HAMILTON, 2009, le 7 octobre 2009, à l'Institut de France, Académie des sciences morales et politiques: <https://www.gemass.fr/lMG/pdf/discours_ RB_7oct09_email.pdf>.

-, 2010, La Sociologie comme science, Paris, La Découverte, «Repères ».

- (dir.), 201 I, «La sociologie: science ou discipline?», Commentaire, 16, hiver, p. $1001-1093$.

-, 2012, Croire et savoir. Penser le politique, la morale, le religieux, Paris, PUF, «Quadrige».

- 2013, «Ma traversée dans le monde scientifique», I et 2, avec Brigitte Masson, Commentaire, 142-143, p. 343-348 et p.603-612.

-, BESNARD Ph., CHERKAOUI M. et LECUYER B.-P. (dir.), 1989, Dictionnaire de la sociologie, Paris, Larousse.

- et BOURRICAUD Fr., 1982, Dictionnaire critique de la sociologie, Paris, PUF.

-, BOUVIER A. et CHAZEL Fr., (dir.), 1997, Cognition et Sciences sociales. La dimension cognitive dans l'analyse sociologique, Paris, PUF.

- et CHERKAOUI M., 1999, Central Currents in Sociological Theory. The roots of sociological theory, Thousand Oaks (CA), Sage, 4 vol.

-, CHERKAOUI M., et DEMEULENAERE P., 2003, The European Tradition in Qualitative Research, Thousand Oaks (CA), Sage, 4 vol.

-, CHERKAOUI M. et JEFFREY A., 1997, The Classical Tradition in Sociology:The European Tradition, Thousand Oaks (CA), Sage, 4 vol.

- et FILLIEULE R., 2002 [Ire éd. 1969, Boudon seul], Les Méthodes en sociologie, Paris, PUF, «Que sais-je?».

- et LAZARFELD P., 1965, Le Vocabulaire des sciences sociales, sélection de textes méthodologiques, précédé d'une introduction, Paris, Mouton. 
- et LAZARFELD P., 1966, L'Analyse empirique de la causalité, sélection de textes méthodologiques, précédé d'une introduction, Paris, Mouton.

-, LAZARFELD P., et CHAZEL Fr., 1969, L'Analyse des processus sociaux, sélection de textes méthodologiques, précédé d'une introduction, Paris, Mouton.

BRONNER G. et GEHIN É., 2017, Le Danger sociologique, Paris, PUF.

BUSINO G. et VALADE B. (dir.), 1998, «La valeur de la sociologie de Raymond Boudon», Revue européenne des sciences sociales, XXXVI-II2.

CAZENEUVE J., 1991, «Allocution», in Remise à Raymond Boudon de son épée d'académicien, Paris, Fayard (suivi de la réponse de Raymond Boudon et de la liste des souscripteurs lui offrant son épée).

CHERKAOUI M. et HAMILTON P. (dir.), 2009, Raymond Boudon, a Life in Sociology, Oxford, The Bardwell Press, 4 vol.

COLLINS R., 1995, «Les traditions sociologiques », Enquête, 2, p. II-38.

DEMEULENAERE P., 20II, Analytical Sociology and Social Mechanisms, Cambridge, Cambridge University Press.

DEVINANT A., 1999, Les Grands courants de la pensée sociologique, Paris, Hachette, 2 vol.

DI NUOSCIO E., 1996, Le ragioni degli individui. L'individualismo methodologico di Raymond Boudon, Soveria Mannelli (Catanzaro), Rubbettino.

DUBOIS M., 2000, Premières leçons sur la sociologie de Raymond Boudon, Paris, PUF. HAMLIN C., 2002, Beyond Relativism. Raymond Boudon, Cognitive Rationality and Critical Realism, Londres / New York, Routledge.

HÉRAN Fr., 2013, «In memoriam Raymond Boudon (1934-2013)», Revue européenne des sciences sociales, 5I-2, p.7-II.

HIRSCHHORN M., 2018, «L'école en sociologie: catégorie, objet, étiquette», Revue d'histoire des Sciences Humaines, 32, p. 153-170.

LANGLOIS S., 2008, «Trente ans de sociologie en France», Commentaire, 31-121, p. 349-359.

MORIN J.-M., 2006, Raymond Boudon. Un sociologue classique, Paris, L'Harmattan. 
SCOPPETTUOLO A., 2010, Etica economica e teoria dell'azione. Ragione, individuo e società in R. Boudon, Soveria Mannelli (Catanzaro), Rubettino.

SIMMEL G., 1984, Les Problèmes de la philosophie de l'histoire, Paris, PUF.

SMELSER N.J. et BATES P.B. (dir.), 200I, International Encyclopedia of the Social and Behavioral Sciences, New York, Elsevier, 26 vol.

VANNIER P., 1999, «Un laboratoire pour la sociologie : le Centre d'études sociologiques (1946-1968) ou les débuts de la recherche sociologique en France», Thèse de doctorat de sociologie, Université Paris Descartes.

VAUTIER Cl., 2002, Raymond Boudon. Vie, Oeurres, Concepts, Paris, Ellipses. 